\title{
Frontières
}

\section{Un parcours poétique pour la Résistance}

\section{Armelle Chitrit}

Volume 19, numéro 1, automne 2006

Enjeux politiques et mort

URI : https://id.erudit.org/iderudit/016645ar

DOI : https://doi.org/10.7202/016645ar

Aller au sommaire du numéro

Éditeur(s)

Université du Québec à Montréal

ISSN

1180-3479 (imprimé)

1916-0976 (numérique)

Découvrir la revue

Citer ce document

Chitrit, A. (2006). Un parcours poétique pour la Résistance. Frontières, 19(1), 99-104. https://doi.org/10.7202/016645ar d'utilisation que vous pouvez consulter en ligne.

https://apropos.erudit.org/fr/usagers/politique-dutilisation/ 


\section{Un parcours poétique pour la Résistance}

\section{Il est un monde entre les mondes contre lequel s'appuie la parole;}

le monde du poème, qui se façonne sans aucun doute, naissant aussi de celui qui nous fait naître et disparaître;

un temps entre les temps pour repousser les limites du dicible;

un temps d'or là où la parole d'argent ne trempe alors ni dans l'innocence ni dans l'horreur;

un temps où le sublime survient, se fait parlant dans la voix du poète,

poème et parole rendant ainsi sensible ce temps entre les temps.

CHITRIT, 1996

\section{Armelle Chitrit, \\ poète et dramaturge.}

À l'ombre de nos pas perdus, se pose aujourd'hui encore la question bien humaine de Résister. Le parcours commémoratif présenté ici propose une réponse possible entre les temps qui nous bousculent. Il réunit poèmes, témoignages vivants et lieux de mémoire autour de la Résistance. Bien qu'il s'y réfère explicitement, il ne se limite ni à un lieu ni à une époque donnés. Créé pour les rues du $1^{\mathrm{er}}$ arrondissement de Lyon, dans le cadre des balades urbaines ${ }^{1}$, il peut s'agencer en divers lieux et formats et dans une perspective d'ouverture sur le présent.

Janvier 2006. Moment venu? moment voulu? ce qui guide nos choix ne s'élucide pas toujours totalement. Dix ans après mon Desnos (Chitrit, 1996), trente ans après le Front populaire, au centenaire de la naissance de mon père (lequel en avait gardé la lumière), le défi s'imposait de réaliser ce temps entre les temps. Invités: Luc Chambon, comédien, petit-neveu de Roger Rastignac, s'est pris au jeu de dire des poèmes avec moi puis Jean-Baptiste Lefebvre, et tous les témoins et passeurs que je remercie: Robert Vasserot, Lucienne et Charles Bossis, Michel Menaché, Claire Claudel, l'équipe de l'Église protestante réformée dans l'héritage du pasteur Roland de Pury... Sans pouvoir les nommer tous, je les remercie ainsi que les équipes du Labo de lettres, du musée Gadagne et du centre de la Résistance de m'avoir encouragée; de ne pas m'avoir laissée seule à dégager ces voix qui veillent dans l'obscurité contre la barbarie.

\section{"SUR L'AIR DE JE ME SOUVIENS ": UNE POÉSIE EN VOIX OFF}

Poursuivie par le fantôme de «ce cri qu'on ne peut pas mettre dans un poème parfait» (Fondane, 1984), une autre devise me hantait: "La violence, c'est pas toujours frappant mais ça fait toujours mal! ${ }^{2} \gg$ Que faire? Comment faut-il faire? Faut-il alors se faufiler? Continuer sa route sur la pointe des pieds? Je crois qu'on peut au moins donner au chant une chance. Il libère, il témoigne ${ }^{3}$ aussi. Tout comme dans Poésie en forme d'espoir ${ }^{4}$, il fallait braver un certain silence, briser la glace, et fi de monuments, construire des moments de paroles. Au cœur du projet, il y a eu ce film ${ }^{5}$ pour lequel j'ai écrit, dans lequel j'ai tourné, qui s'est mis à me parler un langage clair : prendre la poésie en cours de route. Par l'exemple simple d'une voix off, je la mêle au possible du dire, à nos pas, au quotidien, pour pouvoir autrement dénoncer, prendre conscience, partager...

\section{DE LA PETITE HISTOIRE À LA GRANDE}

Dès la première, des inconnus sont venus troubler leurs certitudes, avec leur vague désir et leur curiosité immense, ils m'ont rassurée sur la pertinence de cette mise en situation ${ }^{6}$. Ce parcours a certainement le mérite d'articuler la petite histoire à la grande.

En creux de cette histoire

il y en a beaucoup d'autres

Et dire que ça pourrait s'arrêter là

qui ne s'écrivent pas.

Notre mémoire est un désert

Et dire que ça pourrait s'arrêter là

une absence de fin 7 .

Entre les rues et dans les traboules ${ }^{8}$ si typiques des Pentes de la Croix-Rousse ${ }^{9}$, m'ont suivie et précédée ces gens d'écoute et de paroles. Ils ont manifesté une sorte de fraîcheur et de reconnaissance devant ce qui nous semble à tous indicible... Là où la poésie fort heureusement ouvre les bras, se mêle de ce qui ne la regarde pas! Là où ma démarche au Labo de lettres ${ }^{10}$ prend tout son sens. 


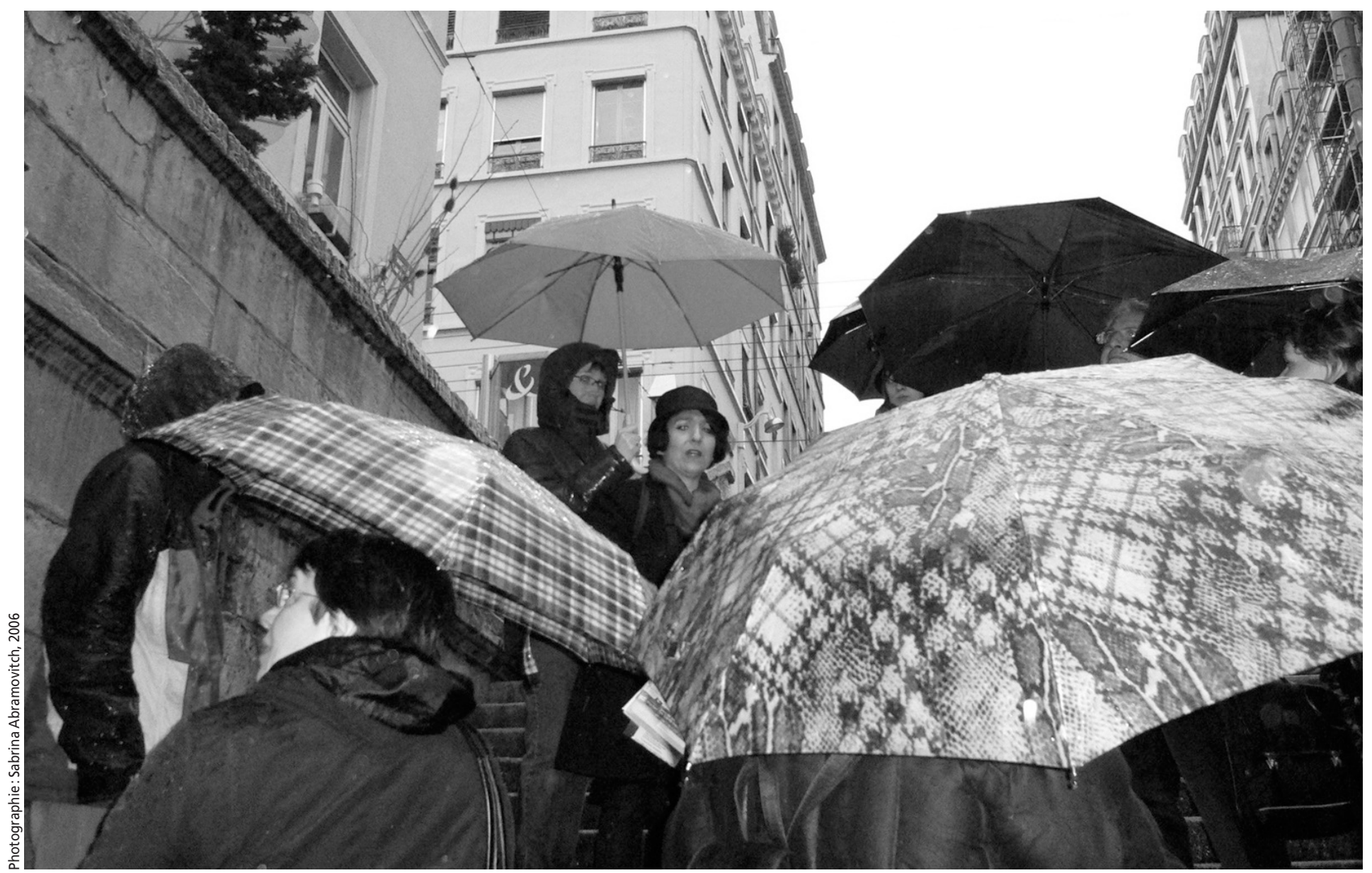

Remontant l'escalier de l'amphithéâtre Trois Gaules, il y a ce long poème scandé que Queneau a conçu en sablier pour nous donner le souffle de gagner un point de vue plus large sur l'humanité:

Tant de sueur humaine

tant de sang gâté

tant de mains usées

tant de chaînes

tant de dents brisées

tant de haines

tant d'yeux éberlués

tant de faridondaines

tant de faridondés

tant de turlutaines

tant de curés

tant de guerres et tant de paix

tant de diplomates et tant de capitaines... ${ }^{11}$

Prenant la rue Burdeau, nous poursuivons en évoquant la mémoire de Lucien Sportisse du Front national de la Résistance (à distinguer du Parti actuel de la honte qui en a récupéré le nom), dont la trajectoire, si proche de celle de mon père, m'a guidée à célébrer tous ceux qui, en Algérie, se sont battus pour un monde meilleur. Je résiste à ce qu'ils fassent aujourd'hui les notes de bas de page! Mon père? Trente ans en 1936; arrêté en 1939 à bord d'un train pour ses opinions politiques, juif et communiste sous Pétain... Après trois ans de prison ferme : vivant! trente-neuf kilos en moins, la peau, les os et quelques bonnes artères pour repartir à zéro.

Cendre des artères du sens

Cendre sang chant

Le pas qui mène aux autres pas $[\ldots]$

Dans la faille d'un plus grand rire

Si le manque venait à gagner

le jugement serait-il premier?

Aurait-on raison de mourir

Ou simplement de s'absenter?

Et pour peu que je n'y sois pas

Cendre chant sang... ${ }^{12}$

J'ai cherché un montage de textes qui touche nos sens et qui, par sa force verbale, de bouche à oreille, symbolise et exerce tout ensemble cette liberté inaliénable avec des pauses critiques, et l'ironie nécessaire à cette sorte de distance dont nous avons aussi besoin. Clin d'œil pour un trop court hommage à Pierre Seghers, poète et éditeur Résistant:

Le cour est fait de mille cordes

Qui se brisent en même temps

Ça fait un bruit épouvantable

Puis plus jamais on ne l'entend

Dans la forêt c'est un grand arbre

les oiseaux y chantent dedans

On en fait du bois pour les tables

Les chiens s'y aiguisent les dents

On dira bien que les tziganes

Font revivre les coeurs perdus

C'est une histoire pour les dames

N'en parlons plus n'en parlons plus ${ }^{13}$.

«Nous ne sommes pas au monde. La vie est ailleurs!» criait Rimbaud, dont la voix douce berce ici et toujours «le Dormeur du 
Val ». On l'aperçoit alors en plongée dans l'angle de l'amphithéâtre des Trois Gaules: «Tranquille. Il a deux trous rouges au côté droit».

\section{RAPPEL: LYON, CAPITALE DE LA RÉSISTANCE ${ }^{14}$}

Durant la Deuxième Guerre mondiale, Lyon a connu des destinées diverses. À partir du 19 juin 1940, 19 jours d'occupation par les troupes allemandes qui se retirent le lendemain de l'établissement de la ligne de démarcation entre Chalon-sur-Saône et Tournus.

Métropole de la zone libre, la Résistance s'organise très tôt à Lyon. Le $1^{\text {er }}$ janvier 1941 a lieu une manifestation publique à l'appel du général de Gaulle. Des démarches individuelles à la constitution de petits groupes, la Résistance prend forme jusqu'à la naissance des premiers mouvements ; s'affirme une volonté d'opposition au régime et de refus de soumission à l'ennemi. L'absence d'occupants (qui n'en ont pas moins leurs observateurs et leurs agents) et la relative tolérance des services de Vichy lui sont plutôt favorables. Entre l'automne 1940 et le printemps 1941 apparaissent plusieurs feuilles et petits journaux clandestins, et les trois grands de la zone sud. On entre aujourd'hui encore dans la librairie alors tenue par monsieur Crozier (proche de Jean Moulin) où l'on imprimait ces journaux distribués lors des réunions clandestines.

À la création du premier journal, Les Petites Ailes, s'ajoute le recrutement de sympathisants. En novembre 1941, le journal clandestin d'Henri Frenay, Vérités, et celui de François Menthon, Liberté, se fondent en un seul, pour donner naissance à Combat, dont le premier numéro paraît en décembre 1941. Combat devient le mouvement le plus important de la zone sud. René Leynaud, responsable des Mouvements unifiés de la Résistance, rejoint ce mouvement. Il est journaliste au Progrès. Un recueil posthume, préfacé par son ami Albert Camus, nous laisse un univers d'une douceur maîtrisée. Voici un extrait de ce merveilleux sonnet qui nous guide dans l'obscurité:

\section{[...]}

J'habite à même l'ombre - ainsi la flamme entière

Pénètre la pensée et meurt sur ses confins

De cire irradiée, car des astres charmèrent

Ma tête tôt éteinte en ses langes de lin.

Vivants je ne le suis sinon qu'en vos poitrines

Réside encor la voix que la mort me ravit

Et redire mon nom me fait l'ombre divine

[...]

(Leynaud, 1947)

Là où il a habité, la rue - anciennement rue de la Vieille monnaie - porte son nom. Minutieusement ici, au numéro 5, la fiction rencontre le réel avec sa lampe chercheuse:

Réside encore la voix que la mort me ravit

$[\ldots]$

(Leynaud, 1947)

En 1942, Lyon, encore en zone libre, est devenue la capitale de la Résistance. La situation évolue radicalement à partir du mois de novembre 1942. En réponse au débarquement allié en Afrique du Nord, l'ennemi, rompant la convention d'armistice, envahit de nouveau la zone sud. Le 11 novembre 1942, la Wehrmacht occupe à nouveau Lyon. La Gestapo s'installe à l'hôtel Terminus situé face à la gare de Perrache. Le Kommando régional (KDS) de Lyon a sous sa férule les départements du Rhône, de la Savoie, de la Haute-Savoie, de la Loire, de la Drôme et de l'Isère. Klaus Barbie s'installe à Lyon fin 1942. En quelques jours, il obtient des succès décisifs contre une résistance lyonnaise parfois victime d'imprudences.
Parmi d'autres, René Leynaud est arrêté place Bellecour, assassiné par les nazis à Villeuneuve, dans l'Ain, à l'âge de 34 ans.

\section{LE POĖTE ET LA NUIT ${ }^{15}$}

Est-ce en ce sens que le poème nous appelle à vouloir en savoir plus? Témoignage d'humanité, garant d'un monde plus juste. Invitation ou devoir? Il parle, appelle à l'éveil plus qu'à la prudence. Le poète ne parle-t-il pas toujours à partir de cette zone d'ombre? Ne change-t-il pas la nature de cette nuit? Le parcours éclaire le jour qui tombe encore avec son lot d'injustices, mais fait aussi passer le poème entre deux portes, du bon côté, avant que le dossier ne se ferme:

Vous qui vivez qu'avez-vous fait de ces fortunes?

Regrettez-vous les temps où je me débattais?

Avez-vous cultivé pour des moissons communes?

Avez-vous enrichi la ville où j'habitais?

Vivants, ne craignez rien de moi car je suis mort.

Rien ne survit de mon esprit ni de mon corps ${ }^{16}$.

Desnos a été déporté à Terezin: il est mort du typhus juste un mois après la Libération. Poète du jour et de la rue, il nous laisse aussi une poésie tout englobée de nuit, une nuit sans mesure. Changeante, peuplée, surpeuplée, cette nuit nous fait passer, insensiblement, du rêveur au veilleur. Elle n'est nuit que pour mieux délivrer le jour. On entend alors chuchoter le poème qui «nous plonge dans l'énigme du temps» (Chitrit, 1996), avec cette «ombre cent fois plus ombre que l'ombre qui viendra et reviendra dans ta vie ensoleillée».

D'hier à aujourd'hui nous avons toujours besoin d'une parole libre et sans complaisance; comment choisir des poèmes dignes de témoigner et de transmettre sur tous les tons un engagement entre notre cause quotidienne et le sens d'une humanité ${ }^{17}$. Je l'ai fait au coup de cœur dans l'idée que chacun construit son parcours et son anthologie. Pourtant s'engager dans la résistance nécessite un acte commun, une parole commune.

Le moment le plus émouvant du parcours s'avère lorsque le mendiant donne ses cartes et que chacun lit à la bougie une strophe de «Liberté » d'Eluard, avant de ressortir des traboules. Il me semble par tous ces éclats de voix, qu'avant d'être écrite, l'histoire nous interpelle, constitue un dialogue sans fin, le mieux et le plus possible informé, pour ne pas se laisser dépasser par ce qui nous dépasse. Grâce à cela, je n'entre plus seule dans cette nuit qui finit par effacer les contours de l'ignorance; grâce à elle nous sentons l'incandescence de l'autre, rendons vivante cette chaîne humaine :

\section{Comme un flot d'oiseaux noirs ils dansaient dans la nuit [...]}

\section{L'ombre en était illuminée: elle flambait L'ennemi s'était endormi ${ }^{18}$}

\section{$[\ldots]$}

Le temps qui se développe à travers une lecture collective nous redonne la nuit comme puissance originelle de toutes ces voix possibles. L'écriture donne voix et visages à une résistance incarnée; aux sentiments se substitue une vérité charnelle, assumée par la parole partagée comme lumière dans l'obscurité. L'autre interlocuteur, ennemi, témoin, sort alors de l'obscurité, du cliché, de l'anonymat.

On ne peut ressortir des traboules sans ressentir la précarité qui nous hante. Du pauvre Rutebeuf à Ossip Mandelstam, j'invente un passage avec le mendiant qui renverse le destin ${ }^{19}$. C'est alors qu'une colère joyeuse éclate et s'empare des «Gueux ${ }^{20}$ » et le cri qui se répand à la barbe des édifices ${ }^{21}$ avant que de pousser la porte, quand elle veut bien s'ouvrir, du numéro 1. 
Ne répond pas à l'appel: Jean Moulin, trahi, torturé puis mort lors de son transfert en Allemagne.

\section{JEAN MOULIN À LYON}

Consacrée capitale de la Résistance par le général de Gaulle le 14 septembre 1944, Lyon occupe une place importante dans l'action de Jean Moulin. C'est dans cette ville, creuset des mouvements de résistance et carrefour géographique, qu'il choisit d'établir son poste de commandement au début de l'année 1942. Le 28 avril, il met en place le BIP (bureau d'information et de presse), «l'agence de presse» de la Résistance qui recueille et transmet les informations à Londres, aux journaux clandestins et aux Alliés, et organise la diffusion de la propagande. Son bulletin d'information est dirigé par Georges Bidault, membre du comité directeur de Combat. Le CGE, qu'il fonde le 3 juillet de la même année, est également basé à Lyon puis s'installe à Paris en avril 1943. Cet organe édite une revue clandestine, Les Cahiers politiques, envoie à Londres des études sur les sujets les plus divers et prépare une refonte administrative pour la Libération. Durant cette période, Jean Moulin travaille seul: sans agent de liaison, sans dactylo; il prépare lui-même ses rendez-vous, distribue en personne l'argent destiné aux officiers de liaison et aux mouvements, chiffre et déchiffre les télégrammes et les rapports reçus et envoyés à Londres. En août 1942, Jean Moulin charge Daniel Cordier de mettre en place, toujours à Lyon, son secrétariat appelé Délégation générale. Organe de commandement et d'exécution, ce groupe de travail autonome, composé de moins d'une dizaine de personnes, se structure peu à peu en plusieurs services: courrier, transmissions, papiers d'identité, trésorerie, etc. Le travail de la Délégation s'effectue dans des conditions précaires et, souvent, sans respecter les règles, bien théoriques, de la sécurité.

À cette époque, les mouvements et les services connaissent un développement accéléré qui exige des liaisons à la fois multiples et rapides vitales pour la Résistance. Daniel Cordier explique: «Lyon présentait cette particularité commode pour les résistants de ne pas avoir de concierges dans les immeubles, qui, en revanche, étaient pourvus de boîtes aux lettres» (Cordier, 1993). Les «courriers» (agents de liaison) effectuent de nombreux déplacements dans la ville, à pied, à vélo ou en tramway. L'organisation de rendez-vous ou de réunions est plus délicate: il faut les préparer longtemps à l'avance, à cause de la lenteur des communications. Il est nécessaire de prévoir un délai: les convocations parviennent aux destinataires qui renvoient leur réponse; en cas de problème, un rendez-vous de «repêchage » s'impose.

Sans le maintien permanent des liaisons entre résistants, la Résistance est paralysée. Chaque fois que des courriers sont arrêtés ou des secrétariats détruits, il faut plusieurs jours pour renouer les liens indispensables et fragiles.

Face aux difficultés et aux dangers de la clandestinité, Jean Moulin s'est organisé une double vie. Du lundi au vendredi, il séjourne à Lyon dans des chambres louées sous un faux nom, notamment place Raspail ou place des Capucins. Chaque fin de semaine, après avoir dîné avec Daniel Cordier près de la gare de Perrache, il quitte la ville par un train du soir en direction du sud, et passé Avignon redevient Jean Moulin avec sa vie officielle.

Le soin qu'il a mis à cloisonner son organisation, la constante vigilance appliquée à ses démarches, le strict respect des règles de comportement, sa réserve qui le met à l'abri des indiscrétions lui permettent de se soustraire aux recherches de plus en plus actives entreprises par les services nazis. Jean Moulin a porté plusieurs pseudonymes : dans la semi-clandestinité, il se fait établir un passeport au nom de Joseph Mercier; en vue de sa mission en France, il porte le pseudonyme de Rex; pour les résistants français, il est connu comme Régis, puis Max. La clandestinité et les incessants déplacements de Jean Moulin à Lyon rendent difficile la reconstitution de son parcours dans la ville. Néanmoins, certains lieux se distinguent plus particulièrement.

\section{RÉSISTER N'EST DONC PAS SUBIR}

Résister n'est donc pas subir en ravalant sa salive. La Résistance ne tient pas de discours; elle se résume à quelques gestes. Non. Il ne s'agit pas de se blinder. C'est l'engagement d'une vie. Il faut certainement du courage, une noblesse de cœur, de l'organisation. Sans doute aussi faut-il du sang-froid et de l'humour, trouver des déguisements et jouer des tours pour déjouer les abus et mettre d'autres âmes au parfum. Les poèmes éduquent à la liberté; ils montrent un rapport au monde dont le langage enchante la diversité au-delà des peurs, au-delà de soi-même. Mais suivant les positions d'énonciation, ils pourraient tout aussi bien servir une propagande. Les parcours protègent une situation inédite de ce côté-là et je crois que c'est cela qui fait leur succès.

Il y a une position nécessaire au-delà des discours pour rallier nos actes à nos valeurs et vivre pour défendre des causes justes. Prenant la question à l'envers, s'agissant de nommer les abus d'une société injuste qui doivent nous maintenir actifs dans notre résistance, notamment la tyrannie, le fanatisme, le règne de la peur, l'oppression arbitraire, la négation de l'individu au profit d'un destin national supérieur, la manipulation de l'information, la propagande... pourrions-nous répondre à l'instar d'Elie Wiesel: «Je respecte l'étranger en l'autre ; c'est l'étrangeté de l'étranger qui me touche» pour quérir l'universalité, le libre arbitre, le doute, la connaissance, la mémoire et par-dessus tout, le respect de l'autre. Il s'agit de combattre non pas "l'intolérance», mais "l'indifférence», un mal qui, selon lui, constitue l'envers de toutes les valeurs positives : le bien, l'amour, la justice, la liberté ${ }^{2}$. Dans mes ateliers, nous jardinons des strophes à la suite du poème d'Éluard:

\section{$[\ldots]$}

Sur le sable et sur la neige

J'écris ton nom.

Mais encore...

\section{ÊTRE JUIF SOUS L'OCCUPATION}

À partir de l'automne 1940, l'adoption d'un train de mesures par l'administration militaire allemande et l'État français exclut peu à peu les Juifs de la communauté nationale. Définis sur la base d'une appartenance raciale ${ }^{23}$, les Juifs n'ont plus le droit d'exercer de responsabilités politiques ni de fonctions dans l'administration publique. L'élargissement de l'éventail des métiers qui leur sont interdits rend d'autant plus difficiles les conditions de survie de beaucoup d'entre eux. Le 22 octobre, la mention «juif» apposée sur les cartes d'identité accentue encore leur marginalisation et permet à l'administration de les identifier.

La création, le 29 mars 1941, du Commissariat général aux questions juives marque la volonté d'appliquer méthodiquement la législation antijuive. Dès mai 1941, les autorités allemandes, avec le concours de la préfecture de police, organisent des rafles visant exclusivement des hommes juifs, nationaux ou étrangers. De son côté, Vichy aggrave la condition des Juifs par un second statut en date du 2 juin 1941, imposant un recensement en zone sud, leur interdisant le droit d'exercer la quasi-totalité des professions, ainsi que l'accès aux lieux publics. Chaque jour, les mesures discriminatoires s'alourdissent, excluant les Juifs de toute vie sociale, les isolant du reste de la population française.

L'année 1942 est marquée par la détermination des autorités à déporter massivement les Juifs de France. Le 29 mai, les Allemands imposent, en zone occupée, le port de l'étoile jaune à tous les Juifs français âgés de plus de six ans. Arrestations et rafles se succèdent: 
les 16 et 17 juillet, près de 13000 Juifs de la région parisienne sont arrêtés au cours de la rafle du Vél d'Hiv.

Sur les 330000 Juifs vivant en France fin 1940, près de 76000 ont été déportés, et seuls 2500 d'entre eux sont revenus.

\section{LA SITUATION À LYON}

À la veille de la Deuxième Guerre mondiale, la communauté israélite de Lyon compte entre 3000 et 4000 membres. Il s'agit principalement de commerçants et d'artisans modestes, républicains et patriotes, proches d'Édouard Herriot et de Justin Godard. Quant à la communauté juive de Villeurbanne, elle est constituée de réfugiés polonais et allemands. Enfin, des immigrés turcs forment un petit groupe à Saint-Fons.

Dès juin 1940, une part importante des Juifs d'Alsace-Lorraine ${ }^{24}$ et nombre de ceux de la zone occupée cherchent à fuir l'emprise de l'occupant. Portés par la conviction que le gouvernement de Vichy assurera leur protection, ils se réfugient à Lyon, venant grossir sensiblement la communauté juive ${ }^{25}$ de la ville. Lors de l'établissement des Allemands à Paris, une partie des organisations juives se replie également à Lyon, notamment le Consistoire central qui s'installe rue Boissac, entre la place Bellecour et la grande synagogue du quai Tilsitt.

Le 20 juillet 1941, les murs de la ville sont placardés d'affiches préfectorales prescrivant le recensement de « tous les Juifs conformément aux termes de la loi du 2 juin $1941 »$. Si la législation antisémite est appliquée de manière rigoureuse, la communauté juive de Lyon n'en bénéficie pas moins du soutien de la population, dont l'hostilité de plus en plus marquée aux mesures antijuives irrite la direction régionale du Commissariat général aux questions juives. À partir d'août 1942, les événements se précipitent: dans la nuit du 20, une première rafle vise les Juifs étrangers. Fin 1942, avec l'installation de la section « antijuive » de la Gestapo, la répression s'intensifie et les rafles se multiplient. Dans ce contexte, organisations sociales ou caritatives juives, chrétiennes ou laïques organisent différentes actions pour sauver les Juifs, et notamment les enfants, de la déportation. Le petit grenier de l'église protestante de la rue Lanterne abritait des enfants qui passaient la frontière pour gagner la Suisse, pays d'origine du pasteur Roland de Pury, arrêté puis condamné pour ses activités résistantes. Un refrain d'Anne Sylvestre nous y ramène:

\section{Moi j'ai le cour tout barbouillé}

Quand vous parlez du petit grenier

Benjamin Fondane, d'origine roumaine, immigre en France entre les deux guerres. Poète, critique et cinéaste, il refuse l'indigne prudence de porter l'étoile et de raser les murs; refuse l'intervention de Jean Paulhan en sa faveur qui ne peut être obtenue pour sa sœur, perd l'espoir de trouver refuge en Argentine. Dans ce poème, on sent que rien ne «le »protège de l'épouvantable fin (Auschwitz Birkenau, 1944):

C'est à vous que je parle, homme des antipodes,

Je parle d'homme à homme,

avec le peu en moi qui demeure de l'homme

avec le peu de voix qui me reste au gosier

mon sang est sur les routes, puisse-t-il

ne pas crier vengeance!...

laissez-moi vous parler avec ces mêmes mots que nous eûmes en partage

Il en reste peu d'intelligibles!

Un jour viendra, c'est sûr, de la soif apaisée, nous serons au-delà du souvenir, la mort aura parachevé les travaux de la haine je serai un bouquet d'orties sous vos pieds, alors, et bien, sachez que j'avais un visage comme vous.

\section{$[\ldots]$}

\section{Et pourtant non!}

je n'étais pas un homme comme vous,

vous n'êtes pas nés sur les routes,

[...]

vous n'avez pas erré de cité en cité, traqués par les polices,

Vous n'avez pas connu les désastres à l'aube,

Les wagons de bestiaux

Et le sanglot amer de l'humiliation, accusés d'un délit que vous n'avez pas fait du crime d'exister [...]

Un jour viendra, sans doute, quand ce poème lu se trouvera devant vos yeux. Il ne demande rien! Oubliez-le oubliez-le! Ce n'est

qu'un cri, qu'on ne peut pas mettre dans un poème parfait, avais-je donc le temps de le finir?

Mais, quand vous foulerez ce bouquet d'orties

qui avait été moi dans un autre siècle

en une histoire qui vous sera périmée, souvenez-vous seulement que j'étais innocent et que, tout comme vous, mortels ce jour-là, j'avais eu, moi aussi, un visage marqué par la colère, par la pitié et la joie.

Un visage d'homme, tout simplement ${ }^{26}$ !

\section{LA RAFLE DE LA RUE SAINTE-CATHERINE27}

Le 9 février $1943^{28}$, la Gestapo de Lyon, sous la direction de Klaus Barbie, organise une rafle dans les locaux du Comité lyonnais de l'Union générale des Israélites de France (UGIF), situés au deuxième étage du 12 , rue Sainte-Catherine.

Un parcours sur les traces de leurs pas change notre angoisse en connaissance. Les habitants s'arrêtent pour en savoir plus, se joignent à la discussion. Dans cette petite rue mal famée derrière les Terreaux, nous évoquons entre autres la mémoire de Simon Badinter.

Composé d'une dizaine d'Allemands en civil et de quelques soldats SS, le commando de la Gestapo investit les lieux en milieu de matinée et procède à l'arrestation d'une trentaine de personnes. Durant plusieurs heures, les agents de la Gestapo mettent en place une souricière ${ }^{29}$, interceptant les personnes qui s'y présentent. Quatre-vingt-six Juifs sont arrêtés, mais deux d'entre eux parviennent à s'échapper ${ }^{30}$.

En soirée, les prisonniers sont emmenés au fort Loyasse, la prison de Montluc étant saturée. Deux ${ }^{31}$ d'entre eux réussissent à s'en échapper. Les quatre-vingt-deux autres sont transférés à Drancy ${ }^{32}$ et déportés à Auschwitz ${ }^{33}$. Il n'y aura que deux survivants. Devant le marbre commémorant l'horreur, d'une petite voix de mère éteinte, je dis cette curieuse berceuse de Pierre Morhange:

Mon bel enfant en habit bleu

Te voilà bien vêtu de velours angoissant

\section{$[\ldots]$}

Mon bel enfant en habit de fumée

Vous ne m'avez pas dit si je peux me tourner ${ }^{34}$

Des documents identifient clairement Klaus Barbie comme étant l'initiateur de cette opération de la rue Sainte-Catherine.

\section{CELA NE S'ARRÊTERA PAS !}

Ce parcours reflète l'exigence d'une voix et d'une rencontre fécondant des temps veilleurs pour un temps meilleur. Il signifie l'expérience de la diversité du réel et sa dissolution active dans la parole poétique qui retourne l'informer. Le temps poétique 
dialoguant ainsi avec l'Histoire guette l'inattendu, l'inconnu, oxygène la mémoire et apprivoise des possibilités. Ponctuer notre route de regards signifiants pour continuer: «le pas qui mène aux autres pas ${ }^{35}$ »

Grâce au rythme, au travail d'improvisation, à l'écoute du public, chaque création de parcours poétique issu de multiples rencontres devient la concentration de leur mouvement dans l'espace. Inutile de dire que la rue, les places, les marchés, les souterrains, larges ou escarpés, les sentiers, regorgent de possibles dans cette architecture particulièrement propice à l'expérience d'une poétique du sujet, de l'espace et du temps.

Tout devient possible, tous publics confondus. Grâce à cette voix inaliénable rendue sensible lors de la médiation, l'interlocuteur n'est jamais laissé pour compte: il emboîte le pas, déjouant avec moi ce qui aurait pu rester routine, indifférence. Mais il s'agit de guetter le moment de grâce comme un chasseur d'une autre sorte!

Nous terminons près du Moulin Joli, café qui se trouve à l'angle des Terreaux, là où fut assassiné Antoine Fonlupt, militant des jeunesses communistes ayant organisé la base des maquis de la vallée d'Azergues avant de rejoindre Lyon où il dirige le groupe des Cordeliers.

Le parcours verbal fait une boucle, note de doute ou d'espoir, accrochée à cette lucidité...
Nous avons peut-être le temps
Encore d'être, et d'être justes.
D'une manière provisoire
La vérité est morte hier,
Cela tout le monde le sait
Bien que chacun le dissimule
Elle n'a point reçu de fleurs
Elle est morte et nul ne la pleure 36 .

\section{Bibliographie}

CORDIER, Daniel (1993). Jean Moulin, l'inconnu du Panthéon, Paris, Jean-Claude Lattès.

CHITRIT, Armelle (2007). Kanutshuk, Lyon, Jacques André Éditeur.

CHITRIT, Armelle (1996). Robert Desnos, le poème entre temps, Lyon, Presses universitaires de Lyon.

DESNOS, Robert (1944). Calixto, suivi de Contrée, Paris, Gallimard.

FONDANE, Benjamin (1984). "Préface en prose. L'Exode Super flumina Babylonis », $L e$ Mal des Fantômes, Toulouse, Patrice Thierry.

LEYNAUD, René (1947). Poésies, Lyon, Gallimard, publication posthume grâce à Francis Ponge.

MARTIN, Hélène (1996). Hélène Martin chante les poètes, EPM. Compilation de chansons enregistrées entre 1962 et 1983, avec la voix de Laurent Terzieff.

\section{Notes}

1. À la demande de l'élu, ces balades sont organisées par le musée Gadagne, musée d'histoire de la ville de Lyon, mandatant divers partenaires dont le Labo de Lettres.

2. Titre d'une campagne de communication sociétale sur la violence conjugale du ministère de la Santé et des Services sociaux du Québec. Voir : www.aimersansviolence.com

3. Mes recherches postdoctorales à l'UQAM dans les équipes de Pierre Ouellet et Régine Robin ont porté sur la poésie comme témoignage notamment des génocides avec un certain nombre de publications que je tarde à rassembler pour un deuxième essai.

4. En résonance avec la Marche mondiale des femmes, au Centre d'éducation et d'action des femmes, dans les écoles de Montréal et enfin au module d'enseignement «Violence et Société » de l'Université de Montréal, 19992000. La devise du Québec étant «Je me souviens ", un devoir de mémoire s'impose pour toutes les sortes de résistance.

5. «Cela s'arrête là », poèmes en voix off, Armelle Chitrit, dans Lise Bonnenfant, De l'ombre à la lumière, Vidéo Femmes, 2000. Ce film articule des témoignages de femmes aux paroles artistes et à diverses commémorations dont celle du massacre de 14 jeunes femmes à l'École polytechnique de Montréal, le 6 décembre 1989.

6. Il s'agit pour l'instant principalement du public des balades urbaines de la ville de Lyon, organisées par le musée Gadagne.

7. «Cela s'arrête là » dans Kanutshuk (2007). Aussi poèmes en voix off, Armelle Chitrit, dans Lise Bonnenfant, De l'ombre à la lumière, Vidéo Femmes, 2000.

8. À Lyon, on appelle traboule le passage qui traverse un pâté de maisons.

9. Quartier poulaire et historique des Canuts, ouvriers de la soie.

10. Le Labo de lettres (Montréal-Lyon, depuis 1998) s'engage à développer une connaissance du monde par la recherche, la création et la transposition de poèmes. L'association privilégie les rapports entre théorie et création, l'articulation avec divers publics, notamment dans les parcours. Elle produit pour une réflexion entre les arts et les sciences, notamment dans les derniers forums du festival Label Bêtes au Muséum de Lyon/musée des Confluences, septembre 2006.

11. Raymond Queneau, «Tant de sueur humaine», dans Martin, 1996.

12. «Le pas qui mène aux autres pas », dans Chitrit, 2007.

13. Pierre Seghers: «Tziganes», dans Martin, 1996.

14. Document de travail du Centre de la Résistance et de la Déportation de Lyon.

15. Je développe cette "nuit superlative» dans La Nuit en question(s), actes du colloque de Cerisy, éditions de l'Aube, 2005.

16. «L'Épitaphe», Contrée, dans Desnos (1944).
17. Voir Frontières, vol. 9 , $\mathrm{n}^{\circ} 3$, p. 60-61, la recension que je fais du livre de Rachel Ertel (1993). Dans la langue de personne. Poésie yiddish de l'anéantissement, Éditions du Seuil, Librairie du $\mathrm{XX}^{\mathrm{e}}$ siècle, 222 pages.

18. Paul Eluard, "Pour ne plus ne plus être seuls ».

19. Ce poème se trouve dans Kerns, recueil de poèmes publié chez Jacques André Éditeur.

20. De Léon Gontran Damas (Guyane).

21. Dont l'église de scientologie.

22. Elie Wiesel en conférence, Montréal, 2006, reportage de Yolande Amsallag.

23. Le statut du 3 octobre 1940 définit comme juive «toute personne issue de trois grandsparents de race juive ou de deux grandsparents de la même race, si son conjoint lui-même est juif ».

24. En juillet 1940, l'Alsace-Lorraine est annexée par les Allemands, les Juifs reçoivent alors l'ordre d'expulsion.

25. Il semble difficile de fournir une estimation exacte car les données chiffrées sont très variables: en 1942, le Grand Rabinat dénombre 70000 Juifs dans l'agglomération alors que le Commissariat général aux questions juives en recense 21659. Cette différence s'explique, en partie, par le fait que nombre de Juifs n'ont pas répondu au recensement.

26. «Exode», dans Fondane, 1984.

27. Document du Centre de la Résistance et de la Déportation de Lyon.

28. Un mardi, jour de distribution et de soins gratuits pour les plus nécessiteux.

29. Les hommes de la Gestapo donnent l'ordre aux standardistes de l'UGIF de répondre aux personnes qui appellent sans trahir leur présence et de les encourager à venir de toute urgence au siège du bureau d'entraide.

30. Sous prétexte d'avertir sa mère malade, Léa Katz-Weiss, alors âgée de 16 ans, obtient d'un des agents de la Gestapo de partir contre la promesse de se présenter le lendemain à l'hôtel Terminus. Quant à Eva Gottlieb, assistante-dactylo bénévole à l'UGIF, elle dispose de faux papiers et explique qu'elle est «venue rapporter une partition de Beethoven à une amie». Elle parvient ainsi à ressortir de l'immeuble. Voir: déposition d'Eva Gottlieb, le 20 mai 1987.

31. Le 15 février 1943, un télex signé par Barbie signale l'évasion de deux détenus, Aron Luxembourg et Siegfried Driller, du fort Loyasse, le 11 février en matinée.

32. Deux sont alors libérés.

33. Parmi les victimes figuraient le père de Robert Badinter et celui de la journaliste Annette Kahn, alors réfugiés à Lyon.

34. Pierre Morhange, "Berceuse à Auschwitz» signé le Blessé.

35. Poème publié dans Kerns, pour cette lutte qui consiste à vivre et à se soigner en dépit des menaces qu'impose le cancer.

36. Pablo Neruda, dans Martin, 1996. 